Research Paper

\title{
Association between the SNPs of the TOBI gene and gastric cancer risk in the Chinese Han population of northeast China
}

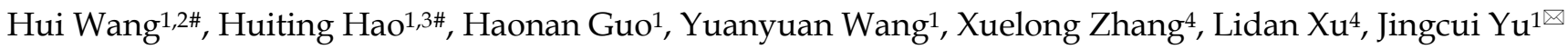 \\ 1. Scientific Research Centre, the Second Affiliated Hospital of Harbin Medical University, Harbin 150081, China \\ 2. Department of Blood Transfusion, the Second Affiliated Hospital of Harbin Medical University, Harbin 150081, China \\ 3. The clinical laboratory, the Tumor Hospital Affiliated to Harbin Medical University, Harbin 150081, China \\ 4. Laboratory of Medical Genetics, Harbin Medical University, Harbin 150081, China. \\ \#These authors contributed equally to this work. \\ $\square$ Corresponding author: Jingcui Yu, M.D., Ph.D. Email: yujingcui@ems.hrbmu.edu.cn \\ (c) Ivyspring International Publisher. This is an open access article distributed under the terms of the Creative Commons Attribution (CC BY-NC) license \\ (https://creativecommons.org/licenses/by-nc/4.0/). See http://ivyspring.com/terms for full terms and conditions.
}

Received: 2017.11.12; Accepted: 2018.01.21; Published: 2018.04.06

\begin{abstract}
The TOBI (ErbB-2,1) gene is an anti-proliferative factor that has the potential to regulate cell growth and encodes a member of the transducer of erbB-2/B-cell translocation gene protein. The association between the polymorphisms of the TOBI gene and gastric cancer (GC) risk is still unclear. In this study, 506 GC cases and 548 healthy controls (HCs) were collected to evaluate the association between the eleven SNPs (rs35220381, rs12950561, rs7221352, rs61482741, rs9303568, rs34700818, rs 12949115, rs9903822, rs 12601477, rs 11656976 and rs4626) of the TOBI gene and GC risk in the population of northeast China. The results showed that there were significant associations of haplotype GCCTTGC, haplotype ATCTTGG, and haplotype GCCACGC with $G C$ risk $(P<0.05, P<0.001$, and $P<0.001$, respectively). The association between $r$ s 12601477 $\mathrm{GA}+\mathrm{AA}$ genotypes and GC risk was significant among individuals older than 58 (adjusted $O R=1.53$, $95 \% \mathrm{Cl}=1.05-2.22, P<0.05$ ). The association between $r s 4626 \mathrm{AG}+\mathrm{GG}$ genotypes and $\mathrm{GC}$ risk was significant among individuals older than 58 (adjusted $\mathrm{OR}=1.54,95 \% \mathrm{Cl}=1.03-2.28, P<0.05$ ). The rs347008 18 CT+TT genotypes were associated with a significantly increased risk of T3-T4 (CT+TT vs $C C$, adjusted $O R=1.71,95 \% \mathrm{Cl}=1.01-2.88, P<0.05)$ and TNM stage $I I(C T+T T$ vs $C C$, adjusted $\mathrm{OR}=2.40,95 \% \mathrm{Cl}=1.27-4.52, P<0.01)$. The rs61482741 CG+GG genotypes were also associated with a significantly increased risk of T3-T4 (CG+GG vs CC, adjusted $O R=1.71,95 \% \mathrm{Cl}=1.01-2.88$, $P<0.05)$ and TNM stage II (CG+GG vs $C C$, adjusted $O R=2.40,95 \% \mathrm{Cl}=1.27-4.52, P<0.01)$. The results suggest that four SNPs (rs12601477, rs4626, rs34700818 and rs61482741) of the TOBI gene play an important role in the occurrence and development of GC in the Chinese Han population of northeast China.
\end{abstract}

Key words: gastric cancer; TOB-1; single nucleotide polymorphism; genetic risk

\section{Introduction}

Gastric cancer (GC) is the fifth most common neoplasia and the third leading cause of cancer-related death worldwide [1]. There were an estimated 951,000 new cases in the world in 2012, and approximately half of the total GC cases were in China [2]. Despite the overall decline in adverse outcomes with the advances in diagnosis and treatment in most of the Western world, GC remains a serious fatal disease and has a poor prognosis throughout Asia, especially in China [3]. GC as a heterogeneous disease shows distinct clinical, epidemiological, and molecular features among tumors arising from the cardia or non-cardia stomach and among the intestinal and diffuse histological 
subtypes [4,5]. Additionally, GC is a multi-step and multi-factorial disease that is influenced by environmental factors, microbial infections, and the host genetic background $[6,7]$.

The TOB1 (transducer of ErbB2,1) gene is a member of the TOB/B cell translocation BTG family, which includes BTG1, BTG2/TIS21/PC3, BTG3/ANA, BTG4/PC3B, TOB1/TOB, and TOB2 [8]. The TOB1 gene is located on chromosome 17q21 and codes for a 45-kDa protein, which was first discovered in the 1990s [9]. As a tumor suppressor, the effect of the TOB1 gene involves many aspects, including anti-proliferation, inhibition of transcription, and the reduction of the migration and invasion of tumor cells in thyroid [10], breast [11], and lung [12] cancers.

We recently identified several allelic deletions on chromosomes 17 and 18 in 45 primary GCs using microsatellite markers for the loss of heterozygosity (LOH). TOB1 lies in one of these regions (17q21.3-22) on the long arm of chromosome $17[13,14]$. Then, we demonstrated that the down-regulation of TOB1 expression and the accumulation of phosphorylated TOB1 promoted carcinogenesis in four GC cell lines and tissue specimens from 97 patients with primary GC [15]. Furthermore, we identified that decreased TOB1 expression and increased phosphorylation of nuclear TOB1 were associated with a malignant tumor phenotype and poor survival in 341 primary GC patients [16].

Recently, increasing numbers of studies have identified that genetic variation plays an important role in the development of most diseases, especially in tumors [17]. Single nucleotide polymorphisms (SNPs), as the most common form of genetic variation, play an important role in the development of GC $[18,19]$. Increasingly more studies have been performed to investigate the association between tumor suppressor gene polymorphisms and GC risk $[18,20]$. A GWAS (genome wide association study) revealed that two SNPs in the PSCA gene were associated with an increased diffuse-type GC risk in a Korean and Japanese population [21]. A meta-analysis indicated that in the P53 codon, 72 polymorphisms might be associated with GC among Asians [22]. However, the association of the SNPs in the TOB1 gene with the risk in malignant tumors (including GC) has not been reported.

TOB1, as a tumor suppressor, encodes a member of the transducer of erbB-2/B-cell translocation gene protein and has the potential to regulate cell growth. However, it is not clear what role the TOB1 gene polymorphisms play in GC risk. Here, we investigated the association between some SNPs in the TOB1 gene and GC risk in a set of 506 GC patients and 548 healthy controls (HCs). Our results suggested that SNPs (rs12601477, rs4626, rs34700818, and rs61482741) in the TOB1 gene are important markers for GC risk in the Chinese Han population.

\section{Material and Methods}

\section{Study population}

A total of 506 unrelated Han Chinese primary GC patients were recruited from The Tumor Hospital Affiliated to Harbin Medical University between January 2015 and June 2016. A total of 548 age- and sex-matched HCs were recruited from The Second Affiliated Hospital to Harbin Medical University during the same period. This study was approved by the ethic committees at local hospitals.

Each participant was interviewed face-to-face by trained interviewers using a standardized questionnaire. The data, including age, gender, family history, native origin, pathological diagnosis, smoking status and alcohol consumption, were collected. Individuals who smoked at least once a day for more than a year were defined as smokers, and the others were defined as non-smokers. Those who consumed alcohol at least once a week for more than a year were defined as drinkers, while the remaining were non-drinkers. The clinical data and demographics of the GC patients and the HCs are summarized in Table 1.

Written informed consent was obtained from each participant. With the permission of the subjects, peripheral venous blood $(5 \mathrm{ml})$ was collected and stored at $-80^{\circ} \mathrm{C}$ in EDTA tubes for DNA extraction.

\section{SNP selection}

The potentially functional SNPs were selected using the NCBI dbSNP database (http://www.ncbi .nlm.nih.gov/snp) and SNPinfo (http://snpinfo.niehs .nih.gov/snpinfo/snpfuc.htm). The criterion applied on the minor allele frequency (MAF) in the NCBI was greater than 0.05 in the Chinese Han population. Ultimately, thirteen SNPs (rs78420930, rs35220381, rs12950561, rs7221352, rs61482741, rs9303568, rs34700818, rs12949115, rs9903822, rs12601477, rs11656976, rs9898809 and rs4626) were selected in our study.

\section{Genotyping}

Genomic DNA was extracted from the blood samples using the Qiagen Blood DNA Mini Kit (Qiagen Inc., Valencia, CA, USA) according to the manufacturer's instructions. The LDR and SNaPshot methods were used to acquire the genotypes of all the SNPs. Genotyping was repeated on a random $10 \%$ of the samples, and the results were $100 \%$ concordant. Ultimately, eleven SNPs (rs35220381, rs12950561, rs7221352, rs61482741, rs9303568, rs34700818, 
rs12949115, rs9903822, rs12601477, rs11656976 and rs4626) were successfully genotyped in 1054 subjects (506 GCs and 548 HCs) and were available for analysis. Two SNPs could not be used to design primers for PCR amplification because of the high GC content.

Table 1. Clinical and demographic characteristics of cases and controls

\begin{tabular}{|c|c|c|c|}
\hline Variables & Case, n (\%) & Control, n (\%) & $P^{a}$ \\
\hline All subjects & $506(100.0)$ & $548(100.0)$ & \\
\hline Age & $23-75$ & $25-87$ & 0.243 \\
\hline Mean ${ }^{b}$ & $59.1 \pm 10.55$ & $58.3 \pm 11.61$ & \\
\hline$\leq 50$ & $107(21.2)$ & $130(23.7)$ & \\
\hline $51-60$ & $156(30.8)$ & $166(30.3)$ & \\
\hline $61-70$ & $169(33.4)$ & $172(31.4)$ & \\
\hline$\geq 71$ & $74(14.6)$ & $80(14.6)$ & \\
\hline Gender & & & 0.363 \\
\hline Male & $371(73.3)$ & $388(70.8)$ & \\
\hline Female & $135(26.7)$ & $160(29.2)$ & \\
\hline Smoking status & & & $<0.000$ \\
\hline Never & $240(47.4)$ & $423(77.2)$ & \\
\hline Ever & $266(52.6)$ & $125(22.8)$ & \\
\hline Drinking status & & & $<0.000$ \\
\hline No & $306(60.5)$ & $419(76.5)$ & \\
\hline Yes & $200(39.5)$ & $129(23.5)$ & \\
\hline Pack-years & & & $<0.000$ \\
\hline 0 & $240(47.4)$ & $423(77.2)$ & \\
\hline$\leq 25$ & $78(15.4)$ & $26(4.7)$ & \\
\hline$>25$ & $188(37.2)$ & 99 (18.1) & \\
\hline \multicolumn{4}{|c|}{ Neoplasia location } \\
\hline GCA & $70(13.8)$ & - & \\
\hline NGCA & $435(86.0)$ & - & \\
\hline Else & $1(0.2)$ & - & \\
\hline \multicolumn{4}{|c|}{ Lauren's classification } \\
\hline Intestinal & $203(40.1)$ & - & \\
\hline Diffuse & $71(14.1)$ & - & \\
\hline Mixed & $81(16.0)$ & - & \\
\hline Else & $151(29.8)$ & - & \\
\hline \multicolumn{4}{|l|}{ TNM stage } \\
\hline I & $87(17.2)$ & - & \\
\hline II & $156(30.8)$ & - & \\
\hline III & $128(25.3)$ & - & \\
\hline IV & $66(13.0)$ & - & \\
\hline Else & $69(13.6)$ & - & \\
\hline \multicolumn{4}{|c|}{ Family history of cancer } \\
\hline Gastric cancer & $42(8.3)$ & - & \\
\hline Other cancer & $63(12.5)$ & - & \\
\hline \multicolumn{4}{|c|}{$\begin{array}{l}\text { Notes: GCA, gastric cardia adenocarcinoma; NGCA, non-gastric-cardia } \\
\text { ademocarcinoma }\end{array}$} \\
\hline
\end{tabular}

\section{Statistical analysis}

Continuous variables with a normal distribution were described as the mean \pm standard deviation and were compared using a Student's t-test. Discrete variables were described as the frequency (percentage) and were compared using the Chi-square $\left(X^{2}\right)$ test. The genotype frequencies for all the TOB1 gene polymorphisms of the controls were tested for Hardy-Weinberg equilibrium by using a Chi-square test. Associations of the genotypes and alleles with the risk of GC were estimated by the odds ratios (ORs) and 95\% confidence intervals (CIs). Linkage disequilibrium (LD) and haplotype analyses were performed with Haploview 4.2 software (http://sourceforge.net/projects/haploview/). P values and ORs with $95 \%$ CIs were calculated using a logistic regression analysis adjusted for age, gender, smoking status, pack-years, and drinking status. All the statistical analyses were performed using the SAS 9.3 software. All P values in the study were two-sided, and $P<0.05$ was considered statistically significant.

\section{Results}

\section{Population characteristics}

A total of 1054 participants (506 GCs and 548 HCs) were enrolled in this study. All the characteristics of the subjects are shown in Table 1. No significant difference between the GC and HC subjects regarding age and gender $(P=0.243$ and $P=0.363$ ) were found, which indicated that the frequency matching was adequate. The mean age was $59.1(59.1 \pm 10.55$ years) for the patients and 58.3 (58.3 \pm 11.61 years) for the controls. However, there was a significant difference $(P<0.0001)$ between the cases and controls regarding smoking status, drinking status, and pack-years. Of the GCs, $70(13.8 \%)$ cases were diagnosed with gastric cardia adenocarcinoma, while $435(86.0 \%)$ cases were diagnosed with non-gastric cardia adenocarcinoma, and $1(0.2 \%)$ was unclear. In term of stage, $87(17.2 \%), 156(30.8 \%), 128$ $(25.3 \%), 66(13.0 \%)$, and $69(13.6 \%)$ cases were classified as TNM stages I, II, III, IV, and else, respectively, according to the 7 th Edition of the American Joint Committee on Cancer (AJCC) [23].

The numbers of participants with no family history of cancer, a history of GC, and a history of other cancers were $401(79.2 \%), 42(8.3 \%)$, and 63 $(12.5 \%)$, respectively.

\section{Association between polymorphisms of the TOBI gene and the risk of GC}

The genotype distributions of the eleven SNPs among the cases and controls and their associations with GC risk are summarized in Supplementary Table 1. The genotype frequencies of all the SNPs of the controls were in accordance with Hardy-Weinberg equilibrium $(P>0.05)$. All the allele frequencies were not significantly different in the case and control groups. Variables including age, gender, smoking, drinking, and pack-years were adjusted for in the subsequent logistic regression analyses. The result showed that none of the eleven SNPs were associated with GC risk in the homozygotes or heterozygotes after adjusting for age, gender, smoking status, pack-years and drinking status $(P>0.05)$. 
Table 2. The frequencies of haplotypes of the TOBI gene in cases and controls

\begin{tabular}{llllll}
\hline Haplotype & Frequency & $\begin{array}{l}\text { Haplotype } \\
\text { frequencies in GC }\end{array}$ & $\begin{array}{l}\text { Haplotype } \\
\text { frequencies in HC }\end{array}$ & $\mathrm{c}^{2}$ & $P$ \\
\hline Block1 & & & & & \\
ACC & 0.566 & 0.553 & 0.578 & 1.353 & 0.245 \\
GCA & 0.254 & 0.257 & 0.251 & 0.087 & 0.768 \\
GCC & 0.110 & 0.117 & 0.104 & 0.980 & 0.322 \\
GTC & 0.063 & 0.066 & 0.061 & 0.184 & 0.668 \\
Block2 & & & & & \\
GCCTTGC & 0.618 & 0.594 & 0.641 & 4.861 & $\mathbf{0 . 0 2 8}$ \\
ATCACGG & 0.236 & 0.228 & 0.244 & 0.772 & 0.380 \\
ACTACAC & 0.061 & 0.058 & 0.063 & 0.252 & 0.616 \\
ACCACAC & 0.035 & 0.039 & 0.032 & 0.792 & 0.373 \\
ATCTTGG & 0.016 & 0.031 & 0.002 & 27.232 & $<0.001$ \\
GCCACGC & 0.011 & 0.022 & 0.001 & 20.638 & $<0.001$ \\
\hline
\end{tabular}

Notes: The bold values indicate statistically significant data

\section{Haplotype analysis and GC risk}

Strong LDs for the SNPs of the TOB1 gene were identified by the Haploview software (Figure 1). Two blocks in the TOB1 gene were found, with four haplotypes in block 1 and six haplotypes in block 2 . The associations between the frequencies of the haplotypes and GC risk are shown in Table 2. The most common haplotype of block 1 was determined as ACC (0.566), followed by GCA (0.254), GCC $(0.110)$, and GTC (0.063). No association between the haplotypes of block 1 and GC risk was observed. The most common haplotype of block 2 was determined as GCCTTGC (0.618), followed by ATCACGG (0.236), ACTACAC (0.061), ACCACAC (0.035), ATCTTGG (0.016), and GCCACGC (0.011). The results showed that there were significant associations of haplotype GCCTTGC, haplotype ATCTTGG, and haplotype GCCACGC with GC risk $(P=0.028, P<0.001$, and $P<0.001$, respectively).

\section{Stratified analysis and GC risk}

We conducted stratified analyses for all the SNPs according to age, gender, smoking status, pack-years, and drinking status, which have potential influences on the genetic effect in the 506 cases and 548 controls of the 1054 participants (Table 3 and Tables S2-S6). As shown in Table 3, for SNP rs12601477, the GA+AA genotypes were associated with a significantly increased risk of GC among individuals older than 58 $(\mathrm{GA}+\mathrm{AA}$ vs $\mathrm{GG}$, adjusted $\mathrm{OR}=1.53,95 \% \mathrm{CI}=$ 1.05-2.22, $P=0.025)$. The rs4626 AG+GG genotypes were also associated with a significantly increased risk of GC among individuals older than 58 (AG+GG vs $\mathrm{AA}$, adjusted $\mathrm{OR}=1.54,95 \% \mathrm{CI}=1.03-2.28$, $P=0.033$ ). No significant association was found between other genotypes of the SNPs and the risk of GC in the stratified analyses by age, gender, smoking status, pack-years, and drinking status.

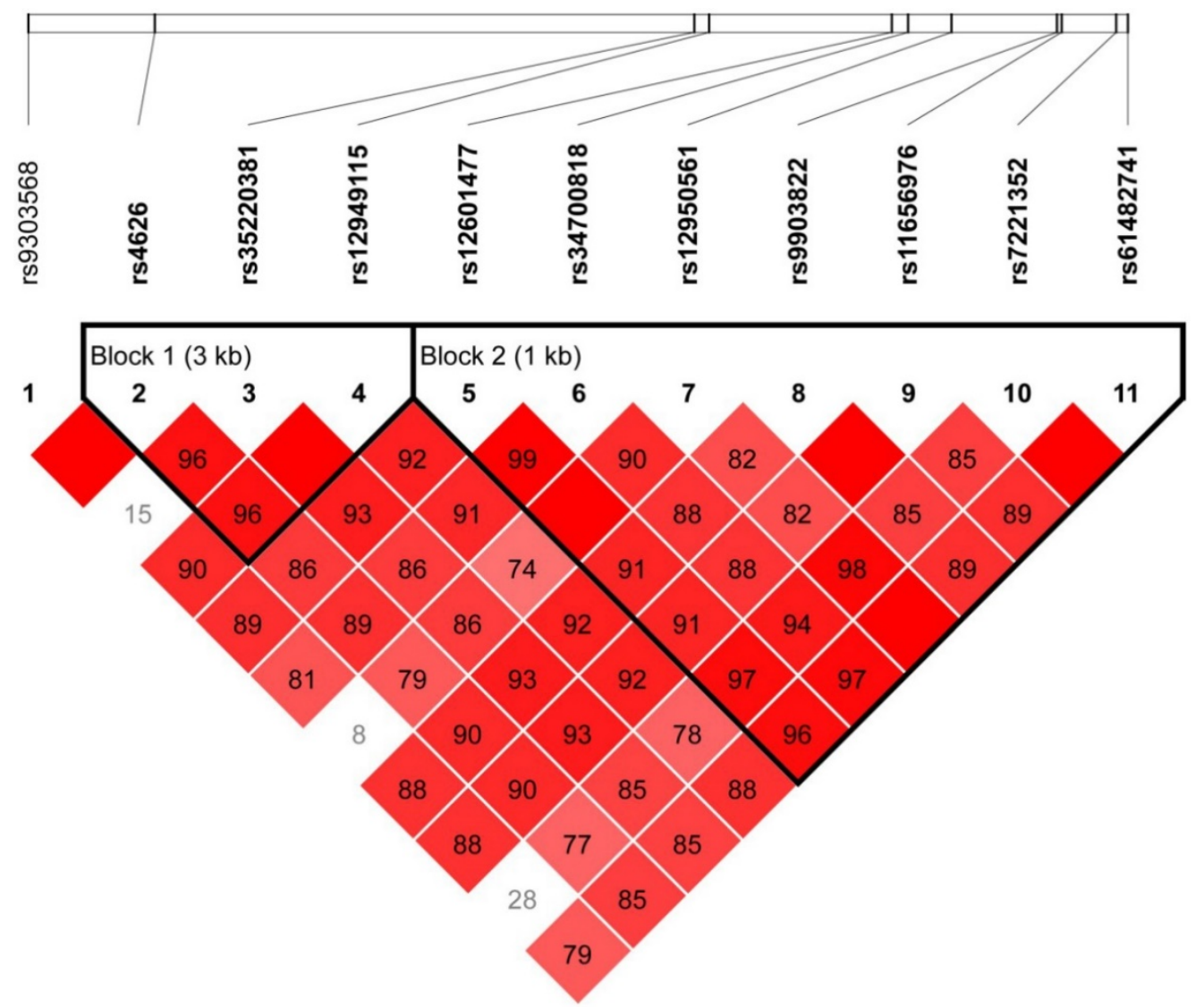

Figure 1. Linkage disequilibrium (LD) map covering TOBI gene 
Table 3. Stratified analyses for TOBI gene rs 12601477 and rs 4626 genotypes in cases and controls

\begin{tabular}{|c|c|c|c|c|c|c|c|c|c|c|c|c|}
\hline \multirow{3}{*}{$\begin{array}{l}\text { Variables } \\
\text { Age }\end{array}$} & \multirow{2}{*}{\multicolumn{2}{|c|}{$\begin{array}{l}\text { rs12601477 } \\
\text { (cases/controls) } \\
\text { GA+AA GG }\end{array}$}} & \multirow[t]{3}{*}{ OR $(95 \% \mathrm{CI})$} & \multirow[t]{3}{*}{$P$} & \multirow{3}{*}{$\begin{array}{l}\text { Adjusted OR } \\
(95 \% \mathrm{CI})\end{array}$} & \multirow[t]{3}{*}{$P^{a}$} & \multirow{2}{*}{\multicolumn{2}{|c|}{$\begin{array}{l}\text { rs4626 } \\
\text { (cases/controls) }\end{array}$}} & \multirow[t]{3}{*}{ OR $(95 \% \mathrm{CI})$} & \multirow[t]{3}{*}{$P$} & \multirow{3}{*}{$\begin{array}{l}\text { Adjusted OR } \\
(95 \% \mathrm{CI})\end{array}$} & \multirow[t]{2}{*}{$P a$} \\
\hline & & & & & & & & & & & & \\
\hline & & & & & & & & & & & & \\
\hline$\leq 58$ & $138 / 158$ & $93 / 107$ & $1.00(0.70-1.44)$ & 0.979 & $0.98(0.67-1.43)$ & 0.913 & $149 / 179$ & $82 / 86$ & $0.88(0.60-1.27)$ & 0.474 & $0.88(0.59-1.30)$ & 0.514 \\
\hline$>58$ & $176 / 153$ & $99 / 130$ & $1.51(1.08-2.12)$ & 0.017 & $1.53(1.05-2.22)$ & 0.025 & $199 / 182$ & $76 / 101$ & $1.45(1.01-2.08)$ & 0.041 & $1.54(1.03-2.28)$ & 0.033 \\
\hline \multicolumn{13}{|l|}{ Gender } \\
\hline Male & $229 / 216$ & $142 / 172$ & $1.28(0.96-1.72)$ & 0.091 & $1.22(0.89-1.66)$ & 0.214 & $253 / 252$ & $118 / 136$ & $1.16(0.86-1.56)$ & 0.344 & $1.15(0.84-1.59)$ & 0.385 \\
\hline Female & $85 / 95$ & $50 / 65$ & $1.16(0.73-1.86)$ & 0.529 & $1.51(0.91-2.53)$ & 0.114 & $95 / 109$ & $40 / 51$ & $1.11(0.68-1.83)$ & 0.678 & $1.42(0.82-2.46)$ & 0.207 \\
\hline \multicolumn{13}{|c|}{ Smoking status } \\
\hline Nonsmoker & $154 / 239$ & $86 / 184$ & $1.38(0.99-1.91)$ & 0.054 & $1.35(0.97-1.87)$ & 0.077 & $172 / 280$ & $68 / 143$ & $1.29(0.91-1.83)$ & 0.146 & $1.26(0.89-1.78)$ & 0.201 \\
\hline Smoker & $160 / 72$ & $106 / 53$ & $1.11(0.72-1.71)$ & 0.632 & $1.18(0.75-1.84)$ & 0.474 & $176 / 81$ & $90 / 44$ & $1.06(0.68-1.66)$ & 0.791 & $1.10(0.69-1.76)$ & 0.674 \\
\hline \multicolumn{13}{|l|}{ Pack-years } \\
\hline 0 & $154 / 239$ & $86 / 184$ & $1.38(0.99-1.91)$ & 0.054 & 1.35 (0.97-1.87) & 0.077 & $172 / 280$ & $68 / 143$ & $1.29(0.91-1.83)$ & 0.146 & $1.26(0.89-1.78)$ & 0.201 \\
\hline$\leq 25$ & $48 / 17$ & $30 / 8$ & $0.75(0.29-1.96)$ & 0.561 & $0.69(0.25-1.92)$ & 0.477 & $49 / 19$ & $29 / 6$ & $0.53(0.19-1.49)$ & 0.230 & $0.42(0.14-1.25)$ & 0.119 \\
\hline$>25$ & $112 / 55$ & $76 / 45$ & $1.21(0.74-1.97)$ & 0.454 & $1.31(0.79-2.17)$ & 0.292 & $127 / 62$ & $61 / 38$ & $1.28(0.77-2.12)$ & 0.345 & $1.38(0.82-2.32)$ & 0.231 \\
\hline \multicolumn{13}{|c|}{ Drinking status } \\
\hline Nondrinker & $185 / 240$ & $121 / 179$ & $1.14(0.84-1.54)$ & 0.391 & $1.16(0.84-1.59)$ & 0.373 & $212 / 279$ & $94 / 140$ & $1.13(0.82-1.55)$ & 0.4436 & $1.13(0.81-1.58)$ & 0.476 \\
\hline Drinker & $129 / 71$ & $71 / 58$ & $1.48(0.94-2.33)$ & 0.087 & $1.49(0.92-2.40)$ & 0.102 & $136 / 82$ & $64 / 47$ & $1.22(0.76-1.94)$ & 0.4065 & $1.26(0.77-2.06)$ & 0.365 \\
\hline
\end{tabular}

Notes: $\mathrm{CI}$, confidence interval; OR, odd ratio

aAdjusted for age age, gender, smoking status, pack-years and drinking status

The bold values indicate statistically significant data

Table 4. Association between TOBI (rs34700818 and rs61482741) genotypes and clinicopathologic characteristics of GC

\begin{tabular}{|c|c|c|c|c|c|c|c|c|}
\hline \multirow[b]{2}{*}{ Variables } & \multicolumn{4}{|c|}{ rs34700818 } & \multicolumn{4}{|c|}{ rs61482741 } \\
\hline & $\begin{array}{l}\mathrm{CT}+\mathrm{TT} \\
(\mathrm{n})\end{array}$ & $\begin{array}{l}\mathrm{CC} \\
(\mathrm{n})\end{array}$ & $\begin{array}{l}\text { Adjusted OR } \\
(95 \% \mathrm{CI})^{\mathrm{a}}\end{array}$ & $P$ & $\begin{array}{l}\text { CG+GG } \\
(\mathrm{n})\end{array}$ & $\begin{array}{l}\text { CC } \\
(\mathrm{n})\end{array}$ & $\begin{array}{l}\text { Adjusted OR } \\
(95 \% \mathrm{CI})^{\mathrm{a}}\end{array}$ & $P$ \\
\hline \multicolumn{9}{|l|}{$\begin{array}{l}\text { Family history } \\
\text { of cancer }\end{array}$} \\
\hline none & 104 & 114 & 1 & & 104 & 114 & 1 & \\
\hline Gastric cancer & 11 & 13 & $0.92(0.39-2.16)$ & 0.844 & 11 & 13 & $0.92(0.39-2.16)$ & 0.844 \\
\hline Other cancer & 15 & 17 & $1.03(0.48-2.18)$ & 0.948 & 15 & 17 & $1.03(0.48-2.18)$ & 0.948 \\
\hline \multicolumn{9}{|l|}{ Tumor size $(\mathrm{cm})$} \\
\hline$<5$ & 71 & 90 & 1 & & 71 & 90 & 1 & \\
\hline$\geq 5$ & 59 & 54 & $1.41(0.86-2.31)$ & 0.176 & 59 & 54 & $1.41(0.86-2.31)$ & 0.176 \\
\hline \multicolumn{9}{|c|}{ Neoplasia location } \\
\hline Non-cardia & 117 & 121 & 1 & & 117 & 121 & 1 & \\
\hline Cardia & 13 & 23 & $0.56(0.27-1.18)$ & 0.125 & 13 & 23 & $0.56(0.27-1.18)$ & 0.125 \\
\hline \multicolumn{9}{|l|}{ Invasion depth } \\
\hline $\mathrm{T} 1-\mathrm{T} 2$ & 35 & 55 & 1 & & 35 & 55 & 1 & \\
\hline T3-T4 & 95 & 89 & $1.71(1.01-2.88)$ & 0.046 & 95 & 89 & $1.71(1.01-2.88)$ & 0.046 \\
\hline \multicolumn{9}{|c|}{ Lymph metastasis } \\
\hline No & 51 & 62 & 1 & & 51 & 62 & 1 & \\
\hline N1/N2/N3 & 79 & 82 & $1.06(0.64-1.75)$ & 0.817 & 79 & 82 & $1.06(0.64-1.75)$ & 0.817 \\
\hline \multicolumn{9}{|l|}{ TNM stage } \\
\hline I & 23 & 46 & 1 & & 23 & 46 & 1 & \\
\hline II & 67 & 54 & $2.40(1.27-4.52)$ & 0.007 & 67 & 54 & $2.40(1.27-4.52)$ & 0.007 \\
\hline III & 40 & 44 & $1.73(0.88-3.43)$ & 0.114 & 40 & 44 & $1.73(0.88-3.43)$ & 0.114 \\
\hline \multicolumn{9}{|c|}{ Lauren's classification } \\
\hline Intestinal & 95 & 108 & 1 & & 95 & 108 & 1 & \\
\hline Diffuse & 35 & 36 & $1.12(0.62-2.03)$ & 0.714 & 35 & 36 & $1.12(0.62-2.03)$ & 0.714 \\
\hline
\end{tabular}

Notes: CI, confidence interval; OR, odd ratio

aAdjusted for age, gender, smoking status, pack-years and drinking status

The bold values indicate statistically significant data

We also conducted stratified analyses based on a family history of cancer, tumor size, neoplasia location, depth of invasion, lymph metastasis, TNM stage and Lauren's classification in 274 patients (Table 4 and Tables S7-S11). As shown in Table 4, the rs34700818 CT+TT genotypes were associated with a significant increase in T3-T4 compared with T1-T2 $(\mathrm{CT}+\mathrm{TT}$ vs $\mathrm{CC}$ adjusted $\mathrm{OR}=1.71,95 \% \mathrm{CI}=1.01-2.88$, $P=0.046)$ and with a significant increase in TNM stage II compared with stage I (adjusted OR=2.40, 95\% $\mathrm{CI}=1.27-4.52, \quad P=0.007)$. The rs61482741 CG+GG genotypes were also associated with a significant increase in T3-T4 compared with T1-T2 (CG+GG vs $C C$, adjusted $\mathrm{OR}=1.71,95 \% \mathrm{CI}=1.01-2.88, P=0.046$ ) and with a significant increase in TNM stage II compared with stage I (CG+GG vs CC, adjusted $\mathrm{OR}=2.40,95 \% \mathrm{CI}=1.27-4.52, P=0.007)$. No association was found between other genotypes of the SNPs and the clinicopathological characteristics of GC.

\section{Discussion}

We previously identified that three overlapping regions (R1-R3) highlighted the association between 
the $\mathrm{LOH}$ on chromosome 17 and GC pathogenesis [13]. Afterward, we narrowed down these intervals using high-density genome scanning and defined five smaller overlapping subregions of LOH (SR1-SR5) in the GC samples. Ultimately, we focused on the TOB1 gene in SR3 (17q21.33), which has not been investigated in GC. Furthermore, our studies demonstrated the down-regulation of TOB1 expression in $75 \%$ of primary GCs and the accumulation of phosphorylated TOB1 in GC cells [15]. Recently, we identified that decreased TOB1 expression and increased nuclear phosphorylated TOB1 were associated with aggressive tumor behavior and a poor prognosis in intestinal type GC. Additionally, TOB1 nuclear retention is critical for its anti-proliferative activity in vitro [16].

Multiple factors, including Helicobacter pylori (Hp) infection [22], nutrition deficiency, a high salt diet, and the chemical carcinogens in tobacco [24, 25], may play an important role, although the etiology and pathogenesis of GC is still uncertain. Furthermore, even if exposed to the same exogenous environmental factors, only a small fraction of people will develop GC, which implies that endogenous genetic variation may also contribute to the individual susceptibility to GC. Recent studies suggest that SNPs may be related to gastric tumorigenesis [19]. Huang $\mathrm{C}$ et al. demonstrated that the CT+TT genotypes of the DACT1 rs863091 polymorphism were significantly associated with a decreased risk of GC in the Chinese Han population, especially in younger individuals and males [18]. DACT1 is a tumor suppressor gene that suppresses tumorigenesis in GC by inhibiting the NF-kB signaling pathway [26]. NF-kB might participate in $\beta$-catenin-mediated target gene expression and eventually results in enhanced tumor growth [27]. TOB1 is a functional anti-oncogene that mainly induces apoptosis and inhibits proliferation, migration and invasion via the activation of Smad4 and the suppression of the $\beta$-catenin-mediated signaling pathways in GC [28].

In the present study, we investigated the associations between the polymorphisms of the TOB1 gene and GC risk in the Chinese Han population. We compared single allele frequencies and a genetic model of the polymorphisms in case-control groups, and no significant differences were found. However, we found that several SNPs of the TOB1 gene were in strong linkage disequilibrium, and haplotype GCCTTGC, haplotype ATCTTGG, and haplotype GCCACGC were significantly associated with GC risk. Moreover, GCCTTGC was the most common haplotype of the TOB1 gene, with a frequency up to $61.8 \%$. Several studies suggest that haplotypes are associated with tumors, such as in TP53, for which the haplotype CCA decreases the risk for GC in a Spanish population $[29,30]$. The polymorphisms included in block 2 were upstream variants of the TOB1 gene, and for the first time, they were associated with cancer risk. We plan to identify their effects in gastric tumorigenesis in future studies.

We also found that the rs12601477 GA+AA genotypes and the rs4626 AG+GG genotypes were associated with a significantly increased risk of GC among individuals older than 58 . This difference may be related to the weaker or disordered immune system in older individuals [31]. TOB1 is expressed in several cell types. It associates with Smad 2 and Smad4 DNA binding and Smad-dependent transcription in T lymphocytes [32]. Increasingly more reports indicate that the TOB1 gene is associated with human immune-related disorders [33]. Low levels of TOB1 may promote an aberrant immune response and affect disease progression [34].

The rs34700818 CT+TT genotypes and the rs61482741 CG+GG genotypes were associated with a significant increase in T3-T4 and TNM stage II. The function of TOB1 involves anti-proliferation, inhibition of transcription and reduction of cancer cell migration, invasion, and metastasis [12]. Gene polymorphisms produced by the replacement, insertion, and loss of a base, which lead to a change in the sequence of the nucleotide, affect the transcription and translation process and ultimately affect the expression of proteins. A GWAS identified that several SNPs are significantly associated with GC. Two GWASs demonstrated that the genetic variant loci in PLCE1 at 10q23 and, for non-cardia GC, at $3 q 13.31$ and 5p13.1 increase the risk of tumors in the stomach [35, 36]. A SNP (rs41274221) in miR-25 regulates the expression of TOB1 by binding with its 3'-UTR region in GC. The mutant genotype promotes cell proliferation and suppresses apoptosis by changing the expression of TOB1 in GC [37].

To date, an increasing number of researches focus on the role of TOB1 in gastric cancerogenesis, which including the localization and expression of the gene[13-15], the mechanisms of inactivation, tumor suppressor involving the participating pathways, and its effect in the cell cycle, etc. [16, 28, 38]. A new study reported that TOB1 gene was contribute to estrogen-independent breast cancer and the interaction effects between TOB1 gene with AKT/mTOR survival signaling [39]. All of the above studies were carried out in cancer tissue or cell lines. Our study is an expansion of the previous work, and it is the first time to investigate the association between TOB1 gene polymorphism and GC susceptibility with molecular markers in peripheral blood. Our results showed that the genotypes of the 
two SNPs (rs34700818 and rs61482741) have significant association with the increased invasion and severity, which suggesting that they impair the inhibition of TOB1. The study provides a direction of predicting the prognosis of the progress of GC and developing some targeting drugs based on these two SNPs. Next, we will continue to enlarge the sample size and to perform functional verification.

There are several limitations in this study, which should be considered. First, partial missing clinical information of the cases, such as data on the TNM stage and Lauren's classification, prevented further analysis. Second, Hp infection is not only an independent but also an important risk factor of GC. The examination of HP infection is not yet a routine examination item. Thus, we did not collect enough information on HP infection for all the subjects, especially for the controls. Third, although our sample size was relatively large, the samples were dispersed in the subgroup analysis and the multilayer analysis. Hence, we will continue to increase the number of GC samples in the next study. Despite these limitations, this is the first study to examine the role of TOB1 gene polymorphisms in the susceptibility to GC. Our findings provide a novel clue for the associations between the SNPs of the TOB1 gene and GC risk in the population of northeast China. Further studies should focus on the effect of the two polymorphisms in the TOB1 gene on gastric tumorigenesis.

In conclusion these results indicated, for the first time, that the four SNPs (rs12601477, rs4626, rs34700818 and rs61482741) of the TOB1 gene are related with GC risk in the Chinese Han population of northeast China. And there are significant association between three haplotypes and GC risk.

\section{Supplementary Material}

Supplementary tables.

http://www.jcancer.org/v09p1371s1.pdf

\section{Acknowledgements}

We thank all individuals who volunteered to participate in this study. This study is supported by the National Natural Science Foundation of China (81372174).

\section{Competing Interests}

The authors have declared that no competing interest exists.

\section{References}

1. Chen W, Zheng R, Baade PD, et al. Cancer statistics in China, 2015. Ca A Cancer Journal for Clinicians. 2016; 66: 115.

2. Ferlay J, Soerjomataram I, Dikshit R, et al. Cancer incidence and mortality worldwide: sources, methods and major patterns in GLOBOCAN 2012. International Journal of Cancer. 2015; 136: E359.
3. Strong VE, Wu A-W, Selby LV, et al. Differences in gastric cancer survival between the U.S. and China. Journal of Surgical Oncology. 2015; 112: 31-7.

4. Kim MA, Lee HS, Yang HK, et al. Clinicopathologic and protein expression differences between cardia carcinoma and noncardia carcinoma of the stomach. Cancer. 2005; 103: 1439-46.

5. Lauren P. THE TWO HISTOLOGICAL MAIN TYPES OF GASTRIC CARCINOMA: DIFFUSE AND SO-CALLED INTESTINAL-TYPE CARCINOMA. AN ATTEMPT AT A HISTO-CLINICAL CLASSIFICATION. Acta Pathol Microbiol Scand. 1965; 64: 31-49.

6. Ang TL, Fock KM. Clinical epidemiology of gastric cancer. Singapore Medical Journal. 2014; 55: 621-8.

7. Daniyal M, Ahmad S, Ahmad M, et al. Risk Factors and Epidemiology of Gastric Cancer in Pakistan. Asian Pacific Journal of Cancer Prevention Apjcp. 2015; 16: 4821-4.

8. Mauxion F, Chen CYA, Séraphin B, et al. BTG/TOB factors impact deadenylases. Trends in Biochemical Sciences. 2009; 34: 640-7.

9. Matsuda S, Kawamuratsuzuku J, Ohsugi M, et al. Tob, a novel protein that interacts with p185erbB2, is associated with anti-proliferative activity. Oncogene. 1996; 12: 705-13.

10. Ito Y, Suzuki T, Yoshida H, et al. Phosphorylation and inactivation of Tob contributes to the progression of papillary carcinoma of the thyroid. Cancer Letters. 2005; 220: 237-42.

11. O'Malley S, Su H, Zhang T, et al. TOB suppresses breast cancer tumorigenesis. Int J Cancer. 2009; 125: 1805-13.

12. Jiao Y, Sun KK, Zhao L, et al. Suppression of human lung cancer cell proliferation and metastasis in vitro by the transducer of ErbB-2.1 (TOB1). Acta Pharmacol Sin. 2012; 33: 250-60.

13. Yu JC, Zhou H, Bai J, et al. Human gastric adenocarcinoma allelotype on chromosomes 17 and 18. Journal of International Medical Research. 2008; 36: 279-88.

14. $\mathrm{Yu} \mathrm{J}$, Zhou $\mathrm{H}$, Jin $\mathrm{Y}$, et al. Three distinct regions of allelic deletion on chromosome 17 involved in sporadic gastric cancer. Hepato-gastroenterology. 2008; 55: 1487-91

15. Yu J, Liu P, Cui X, et al. Identification of novel subregions of $\mathrm{LOH}$ in gastric cancer and analysis of the HIC1 and TOB1 tumor suppressor genes in these subregions. Molecules \& Cells. 2011; 32: 47-55.

16. Guan R, Peng L, Wang D, et al. Decreased TOB1 expression and increased phosphorylation of nuclear TOB1 promotes gastric cancer. Oncotarget. 2017; 8: 75243-53.

17. Park SL, Caberto CP, Lin Y, et al. Association of cancer susceptibility variants with risk of multiple primary cancers: The population architecture using genomics and epidemiology study. Cancer Epidemiol Biomarkers Prev. 2014; 23: $2568-78$

18. Chi H, Wang Y, Hao F, et al. Association analysis of DACT1 genetic variants and gastric cancer risk in a Chinese Han population: a case-control study. Oncotargets \& Therapy. 2016; 9: 5975-83

19. Machado JC, Pharoah P, Sousa S, et al. Interleukin $1 B$ and interleukin $1 R N$ polymorphisms are associated with increased risk of gastric carcinoma. Gastroenterology. 2001; 121: 823-9.

20. Wu GC, Zhang ZT. Genetic association of single nucleotide polymorphisms in P53 pathway with gastric cancer risk in a Chinese Han population. Medical Oncology. 2015; 32: 1-5.

21. Sakamoto H, Yoshimura K, Saeki N, et al. Genetic variation in PSCA is associated with susceptibility to diffuse-type gastric cancer. Nature Genetics. 2008; 40: 730-40.

22. Zhou Y, Li N, Zhuang W, et al. P53 codon 72 polymorphism and gastric cancer: a meta-analysis of the literature. International Journal of Cancer. 2007; 121: $1481-6$

23. Mcghan LJ, Pockaj BA, Gray RJ, et al. Validation of the Updated 7th Edition AJCC TNM Staging Criteria for Gastric Adenocarcinoma. J Gastrointest Surg. 2012; 16: 53-61.

24. Ladeiraslopes R, Pereira AK, Nogueira A, et al. Smoking and gastric cancer: systematic review and meta-analysis of cohort studies. Cancer Causes \& Control Ccc. 2008; 19: 689-701.

25. Palli D. Epidemiology of gastric cancer: an evaluation of available evidence. Journal of Gastroenterology. 2000; 35 Suppl 12: 84-9.

26. Wang S, Kang W, Go MY, et al. Dapper homolog 1 is a novel tumor suppressor in gastric cancer through inhibiting the nuclear factor- $\mathrm{kB}$ signaling pathway. Molecular Medicine. 2012; 18: 1402-11.

27. Schwitalla S, Fingerle AA, Cammareri P, et al. Intestinal tumorigenesis initiated by dedifferentiation and acquisition of stem-cell-like properties. Cell. 2013; 152: 25.

28. Kundu J, Wahab SMR, Kundu JK, et al. Tob1 induces apoptosis and inhibits proliferation, migration and invasion of gastric cancer cells by activating Smad 4 and inhibiting $\beta$-catenin signaling. International Journal of Oncology. 2012; 41: 839-48.

29. Humar B, Graziano F, Cascinu S, et al. Association of CDH1 haplotypes with susceptibility to sporadic diffuse gastric cancer. Oncogene. 2002; 21: 8192-5.

30. Carrera-Lasfuentes P, Lanas A, Bujanda L, et al. Relevance of DNA repair gene polymorphisms to gastric cancer risk and phenotype. Oncotarget. 2017; 140: 35848-62.

31. Zhu H, Yang L, Zhou B, et al. Myeloperoxidase G-463A polymorphism and the risk of gastric cancer: a case-control study. Carcinogenesis. 2006; 27: 2491-6. 
32. Tzachanis D, Freeman GJ, Hirano N, et al. Tob is a negative regulator of activation that is expressed in anergic and quiescent $\mathrm{T}$ cells. Nature Immunology. 2001; 2: 1174-82.

33. Baranzini SE. Role of antiproliferative geneTob1in the immune system. Clinical and Experimental Neuroimmunology. 2014; 5: 132-6.

34. Didonna A, Cekanaviciute E, Oksenberg JR, et al. Immune cell-specific transcriptional profiling highlights distinct molecular pathways controlled by Tob1 upon experimental autoimmune encephalomyelitis. Scientific Reports. 2016; $6: 31603$.

35. Abnet CC, Freedman ND, Hu N, et al. A shared susceptibility locus in PLCE1 at $10 \mathrm{q} 23$ for gastric adenocarcinoma and esophageal squamous cell carcinoma. Nature Genetics. 2010; 42: 764-7.

36. Shi $\mathrm{Y}, \mathrm{Hu} \mathrm{Z}, \mathrm{Wu} \mathrm{C}$, et al. A genome-wide association study identifies new susceptibility loci for non-cardia gastric cancer at $3 q 13.31$ and $5 p 13.1$. Nature Genetics. 2011; 43: 1215-8.

37. Zhou J, Zhou J, Wang W, et al. The polymorphism in miR-25 attenuated the oncogenic function in gastric cancer. Tumor Biology. 2016; 37: 5515-20.

38. Lee HS, Kundu J, Kim RN, et al. Transducer of ERBB2.1 (TOB1) as a Tumor Suppressor: A Mechanistic Perspective. International Journal of Molecular Sciences. 2015; 16: 29815-28.

39. Zhang $\mathrm{Y}$ W, Nasto $\mathrm{R}$ E, Varghese $\mathrm{R}$, et al. Acquisition of Estrogen Independence Induces TOB1-Related Mechanisms Supporting Breast Cancer Cell Proliferation. Oncogene, 2016, 35(13):1643 